\title{
Optimisation of additional coils of a vibration energy harvester using interior point algorithm
}

\author{
Marcin Kulik $^{1, *}$, Mariusz Jagieła ${ }^{1}$ and Bernard Baron ${ }^{1}$ \\ ${ }^{1}$ Opole University of Technology, Faculty of Electrical Engineering, Automatic Contr. and Informatics, Opole, Poland
}

\begin{abstract}
The paper aims at applying the interior point algorithm in optimisation of additional coils of a small resonant electromagnetic vibration energy harvester. The system, which consists of a coreless microgenerator is driven by external vibration through a cantilever-beam spring element. Originally, the system contains only one coil mounted centrally between the moving magnets. It was found that the very low induced voltage can be significantly increased by exploitation of leakage flux outside the magnets using additional coils, whose are optimised for maximum voltage.
\end{abstract}

\section{Introduction}

The system considered in this work, which belongs to the group of electromagnetic vibration energy harvesters [1-3] is shown in Fig. 1.

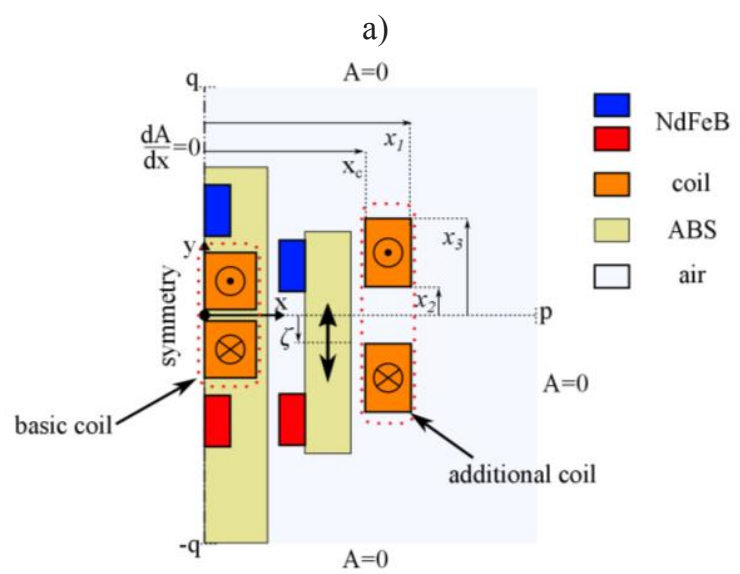

b)

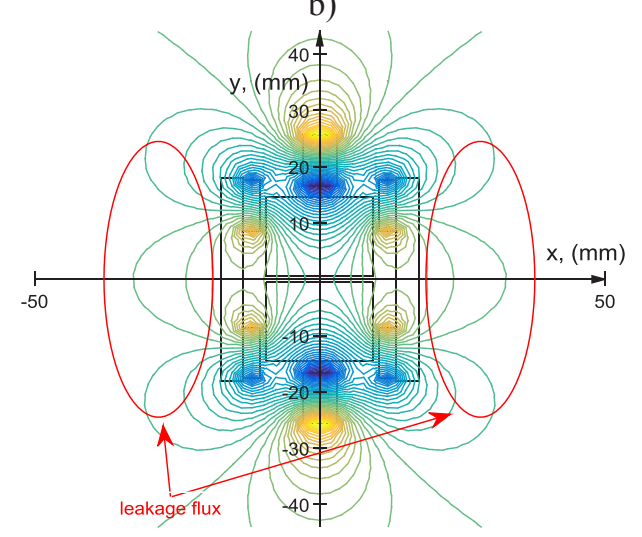

Fig. 1. Considered system: a) schematic diagram b) distribution of magnetic flux lines at displacement of magnets $\zeta=0$.
The distribution of magnetic field in Fig. $1 \mathrm{~b}$ was obtained by analytic solution of the Poisson equation

$$
v_{0} \nabla^{2} A=J_{M}
$$

with $A$ being the magnetic vector potential, $v_{0}$ the reluctivity of air, and $J_{M}$ the equivalent current density due to permanent-magnets. The equation that describes the flux linkage of coil $\Psi$ was derived in [2]. In this work we optimise the maximum value of induced voltage which appears at $\zeta=0$, consequanty the objective function is

$$
f(\boldsymbol{x})=-\left.\frac{\partial \Psi(\boldsymbol{x}, \zeta)}{\partial \zeta}\right|_{\zeta=0}
$$

where (see Fig. 1a) $\mathbf{x}=\left[x_{1}, x_{2}, x_{3}\right]$ is a vector of decision variables. In order to provide feasibility of the design space the follwing constraints are acounted for

$$
g(x) \geq 0
$$

where :

$$
\begin{aligned}
& \boldsymbol{g}=\left[\begin{array}{lllll}
x_{1}-x_{c} & x_{2}-1 & x_{3}-x_{2}-1 & q-x_{2}-x_{3}-1 & \ldots
\end{array}\right. \\
& \ldots p-x_{2}-x_{3}-1 \sqrt{\frac{4 \mathrm{k}\left(x_{1}-\mathrm{x}_{\mathrm{c}}\right)\left(x_{3}-x_{2}\right)}{N \pi}}-0.2 m m \quad \ldots \\
& \left.0.4 m m-\sqrt{\frac{4 \mathrm{k}\left(x_{1}-\mathrm{x}_{\mathrm{c}}\right)\left(x_{3}-x_{2}\right)}{N \pi}}\right]
\end{aligned}
$$

with $x_{c}=18.39 \mathrm{~mm}, p=75 \mathrm{~mm}, q=75 \mathrm{~mm}$ (see Fig. 1a), $k=0.8$ is a coil fill factor by wires, $N=1000$ is the assumed number of turns. The constraints provide that the area of the optimised coils' cross-sections do not exceed the value equal to the $N \cdot d / \mathrm{k}$, where $d$ is the wire diameter. Since $f(x)$ is given analytically its variation could be explored before the optimisation. Fig. 2 presents such the variations with repsect to the two

\footnotetext{
Corresponding author: marcin.kulik@doktorant.po.edu.pl
} 
selected design variables, whilst the third one was set as constant.

a)
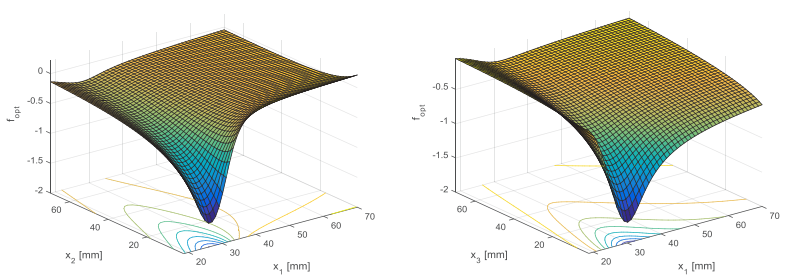

c)

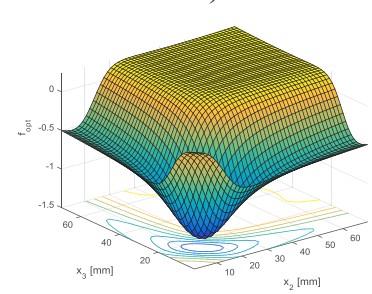

Fig. 2. Variations of objective function: a) vs. $x_{1}$ and $x_{2}$, b) vs. $x_{1}$ and $\left.x_{3}, \mathrm{c}\right)$ vs. $x_{2}$ and $x_{3}$.

\section{Optimisation}

Minimisation of (2) subject to (3) using the interior point (IP) algorithm ensures that neither design variable can leave the feasible region [4].This is possible thanks to introduction of the logarithmic barrier function which modifies the orginal minimisation algorithm to [4]

$$
\begin{gathered}
\min _{\mathbf{x}}\left(f(\mathbf{x})-\mu_{k} \sum_{i=1}^{n_{g}} \ln \left(z_{i}\right)\right) \\
\mathbf{g}(\mathbf{x})-\mathbf{z}=0, \mathbf{z} \geq 0
\end{gathered}
$$

where $\mu_{\mathrm{k}}$ is a barrier parameter which is calculated using formula in [4]. In the IP algorithm the optimisation problem is to find the stationary point of the following Lagrange function

$$
L_{\mu}\left(\mathbf{y}_{k}, \mu_{k}\right)=f(\mathbf{x})-\mu_{k} \sum_{i=1}^{n_{g}} \ln \left(z_{i}\right)+\boldsymbol{\pi}^{\mathrm{T}}(\mathbf{g}(\mathbf{x})-\mathbf{z})
$$

with $\pi$ being the vector of Lagrange multipliers, which is carried out using the Newton-Rhapson method [4].

\section{Results}

The starting points for the algorithm were selected using the Latin Hypercube sampling. From the 50 random solutions the three best ones, which fall into the area restriced by (3), are selected. The results of optimisation for such starting points are given in Tab.1.

Table. 1. Results of optimisation.

\begin{tabular}{|c|c|c|c|}
\hline Starting point $\mathbf{x}_{0}$ & $\begin{array}{c}\text { No. of } \\
\text { iterations }\end{array}$ & $\begin{array}{c}\text { Exec. } \\
\text { time }\end{array}$ & $\begin{array}{c}\text { Solution } \\
\mathbf{x}_{\text {opt }}\end{array}$ \\
\hline$\left[\begin{array}{lllll}20.97 & 4.03 & 0.05\end{array}\right]^{\mathrm{T}}$ & 14 & $2.8 \mathrm{~s}$ & {$\left[\begin{array}{llll}22.51 & 10.22 & 9.75\end{array}\right]^{\mathrm{T}}$} \\
\hline$\left[\begin{array}{llll}22.39 & 7.82 & 3.40\end{array}\right]^{\mathrm{T}}$ & 13 & $2.7 \mathrm{~s}$ & {$\left[\begin{array}{lll}22.51 & 10.22 & 9.75\end{array}\right]^{\mathrm{T}}$} \\
\hline$\left[\begin{array}{lll}28.43 & 11.04 & 15.86\end{array}\right]^{\mathrm{T}}$ & 13 & $2.7 \mathrm{~s}$ & {$\left[\begin{array}{lll}22.51 & 10.22 & 9.75\end{array}\right]^{\mathrm{T}}$} \\
\hline
\end{tabular}

Fig. 3 shows the variations of (2) for the optimal solution as well as for the three other randomly selected initial designs. The most favoured optimal solution (due to high voltage at small displacement) is plotted using solid line. As one can notice the induced voltage was increased by some 20 per cent with respect to the best initial solution.

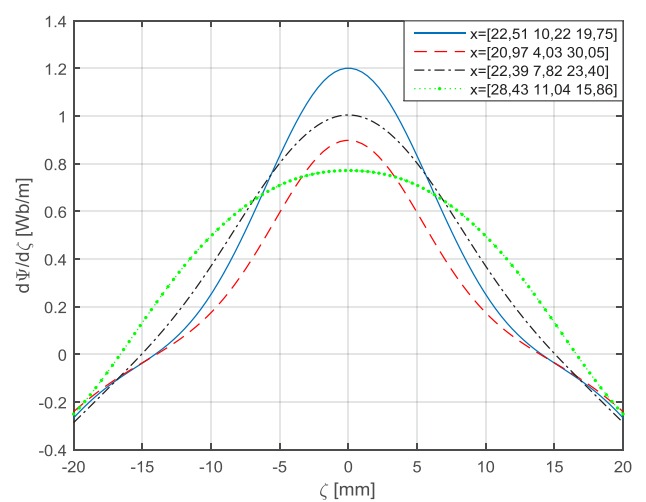

Fig. 3. Variations of flux linkage derivative for initial and optimal designs.

\section{Conclusion}

In this work the application of the interior point algorithm to maximisation of voltage induced in additional coils of a vibration energy harvester was presented. One important advantage of the method, beside a good numerical performance, is that in contrast to other tested local search algorithms, such as Powell, Jives-Hooke, Broyden-Fletcher-Goldfarb-Shanno, and conjugate gradient algorithms, it ensures that during evaluation of the objective function the design variables never leave the feasible region. This is very important advantage from the point of view of optimisation of physical structures, especially when the objective function is determiend from numerical models such as the finite element models.

The results obtained in this works are the basis for development of new system which is currently under construction.

This work was carried out under project 2016/23/N/ST7/03808 of the National Science Centre, Poland

\section{References}

1. P. D. Mitcheson, E. M. Yeatman, G. K. Rao, A. S. Holmes, T. C. Green, Proceedings of the IEEE 96, 9 (2008)

2. M. Jagieła, M. Kulik, Int. Journ. of Applied Electromagn. and Mechanics 51, S1 (2018)

3. H. Lee, M. D. Noh, Y. W. Park, IEEE Trans. Magn. 53, 11 (2017)

4. M. Połomski, B. Baron, Acta Energ. 1, 14 (2013) 